\title{
Quercetin Attenuates D-GaLN- Induced L02 Cell Damage by Suppressing Oxidative Stress and Mitochondrial Apoptosis via Inhibition of HMGB1
}

\section{OPEN ACCESS}

Edited by:

Wei Peng,

Chengdu University of Traditional Chinese Medicine, China

Reviewed by: Ping Yao,

Huazhong University of Science and Technology, China

Marco Emilio Bianchi, Vita-Salute San Raffaele University,

Italy

${ }^{*}$ Correspondence: Qiuyun Zhang zhangqiuyun8202@aliyun.com

Specialty section: This article was submitted to Experimental Pharmacology and Drug Discovery, a section of the journal

Frontiers in Pharmacology

Received: 12 December 2019

Accepted: 17 April 2020

Published: 05 May 2020

Citation:

Fang $P$, Liang J, Jiang $X$, Fang $X$, Wu $M$, Wei $X$, Yang W, Hou W and Zhang Q (2020) Quercetin Attenuates D-GaLN-Induced L02 Cell Damage

by Suppressing Oxidative Stress and Mitochondrial Apoptosis via Inhibition of HMGB1.

Front. Pharmacol. 11:608 doi: 10.3389/fphar.2020.00608

\author{
Peng Fang, Jiajun Liang, Xuejiao Jiang, Xian Fang, Mengli Wu, Xiaoyi Wei, \\ Wenlong Yang, Weixin Hou and Qiuyun Zhang * \\ Beijing Key Laboratory of TCM Collateral Disease Theory Research, School of Traditional Chinese Medicine, Capital Medical \\ University, Beijing, China
}

High mobility group box-1 (HMGB1) plays an important role in various liver injuries. In the case of acute liver injury, it leads to aseptic inflammation and other reactions, and also regulates specific cell death responses in chronic liver injury. HMGB1 has been demonstrated to be a good therapeutic target for treating liver failure. Quercetin (Que), as an antioxidant, is a potential phytochemical with hepatocyte protection and is also considered to be an inhibitor of HMGB1. However, the mechanism of its hepatoprotective effects remains to be characterized. The present study explored whether the hepatoprotective effect of Que antagonizes HMGB1, and subsequent molecular signaling events. Our results indicated that Que protects L02 cells from Dgalactosamine (D-GaLN)-induced cellular damage by reducing intracellular reactive oxygen species (ROS) production and apoptotic responses in the mitochondrial pathway. Immunofluorescence and Western blot assays showed that HMGB1 was involved in D-GaLN-induced L02 cell damage. Further research showed that after transfection with HMGB1 short hairpin RNA (shRNA), cell viability was improved, and intracellular ROS production and apoptosis were suppressed. When co-treated with Que, the expression of HMGB1 was decreased significantly, the expression of proteins in the corresponding signal pathway were further reduced, and the production of ROS and apoptosis were further suppressed. Molecular docking also indicated the binding of Que and HMGB1. Taken together, these results indicate that Que significantly improves DGaLN-induced cellular damage by inhibiting oxidative stress and mitochondrial apoptosis via inhibiting HMGB1.

Keywords: quercetin, high mobility group box-1, D-galactosamine, oxidative stress, apoptosis 


\section{INTRODUCTION}

High mobility group box-1 (HMGB1) is an evolutionarily conserved nuclear DNA-binding protein widely found in eukaryotic cells, which has multiple conflicting functions (both inflammatory and cell protective), depending on its location (Kang et al., 2014; Musumeci et al., 2014). HMGB1 acts as a damage-associated molecular pattern (DAMP) molecule, when it is passively released after cell damage or actively secreted into the extracellular space, it communicates the occurrence of injury and inflammation to neighboring cells via the receptor for advanced glycation end products (RAGE) or toll-like receptor 4 (TLR-4) (Scaffidi et al., 2002; Huebener et al., 2015). HMGB1 contributes to aseptic inflammation and other responses in acute liver injury, playing a key role (Yang et al., 2017). It is also an important hepatocyte DAMP, which regulates specific cell death responses in chronic liver injury (Hernandez et al., 2018). Studies have shown that serum HMGB1 levels in patients with acute or chronic liver failure (ACLF) are significantly higher than those in healthy controls and patients with chronic hepatitis B (CHB) (Hu et al., 2017). Hepatocyte-derived HMGB1 is also involved in liver fibrosis. Blocking HMGB1 can partially prevent the consequences of mouse CCL4-induced liver fibrosis (Zhang et al., 2018). Moreover, the experiment targeting HMGB1 demonstrated it was a good therapeutic target for liver failure (LF) (Yamamoto and Tajima, 2017).
HMGB1 release induced by hepatic ischemic injury involves TLR-4-dependent reactive oxygen species (ROS) production and calcium-mediated signaling (Zhang et al., 2014). Due to the predominant role of hepatocytes in the biotransformation and metabolism of xenobiotics, ROS production constitutes a severe burden in liver pathophysiology in the progression of liver diseases (Klotz and Steinbrenner, 2017). The oxidized HMGB1 mediates apoptosis, and the production of HMGB1 is also a common downstream factor for multiorgan damage caused by apoptosis (Bai et al., 2017; Petrovic et al., 2017).

Quercetin (Que) (3,5,7,3',4'-pentahydroxyflavone) (Figure 1) is a typical flavonol-type flavonoid commonly found in vegetables, fruits, nuts, beverages, and traditional Chinese herbs (Darband et al., 2018). Que has been reported to possess a broad array of biological effects, including antioxidative, antiinflammatory, and anti-apoptotic effects (de Oliveira et al., 2016; Zheng et al., 2017). It is now largely utilized as a nutritional supplement and as a phytochemical remedy for a variety of hepatic diseases like hepatitis, cirrhosis, acute liver failure, alcoholic or non-alcoholic fatty liver disease, and fibrosis (Miltonprabu et al., 2017; Li et al., 2018). Que has exhibited strong defensive effects against apoptosis, inflammation, and ROS generation in the liver of experimental animals exposed to various hepatotoxicants (Zou et al., 2015; Wang et al., 2017).

As an antioxidant, Que is also considered to be an inhibitor of HMGB1 (Li et al., 2016). However, it is not well known if the
A<smiles>O=c1c(O)c(-c2ccc(O)c(O)c2)oc2cc(O)cc(O)c12</smiles>

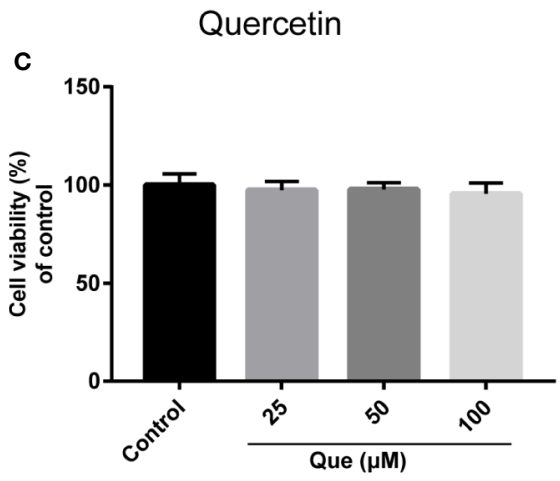

B

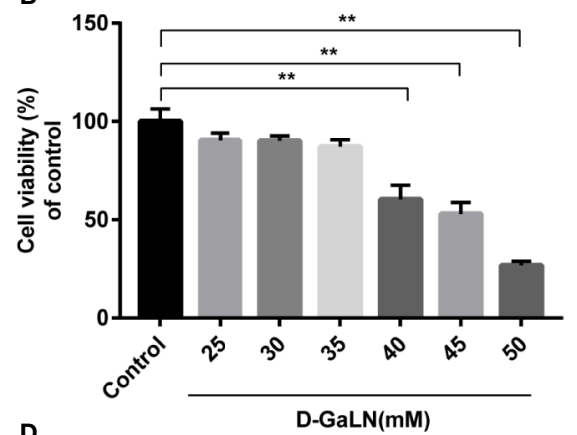

D

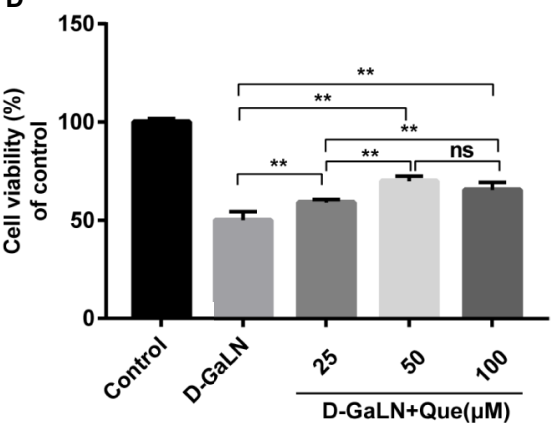

FIGURE 1 | Protective effect of quercetin (Que) on D-galactosamine (D-GaLN)-induced cytotoxicity in L02 cells. (A) The chemical structure of Que. (B) Cells were treated with different concentrations of D-GaLN (25, 30, 35, 40, 45, $50 \mathrm{mM}$ ) (C) or Que (25, 50, $100 \mu \mathrm{M})$ for $12 \mathrm{~h}$. (D) Cells were pre-treated with Que (25, 50, 100 $\mu \mathrm{M})$ for $12 \mathrm{~h}$ and then co-treated with D-GaLN (45 mM) for $12 \mathrm{~h}$. A Cell Counting Kit-8 (CCK8) assay was used to analyze cell viability. Data are presented as the mean $\pm \mathrm{SD},\left({ }^{*} p<0.05,{ }^{* *} p<0.01, \mathrm{n}=6\right) ; " n s "$ indicates not significant $(p>0.05)$. 
hepatoprotective effect of Que occurs through the antagonism of HMGB1 and the ensuing molecular signaling events. Therefore, the aim of this study was to investigate whether Que could protect L02 cells by inhibiting HMGB1, in addition to examining the underlying mechanism of Que, in order to provide a theoretical basis for Que as a hepatoprotective drug targeting HMGB1.

\section{MATERIALS AND METHODS}

\section{Chemicals and Reagents}

Quercetin was obtained from Sigma-Aldrich (St. Louis, USA; cat: Q4951); its purity $\geq 95 \%$. D-Galactosamine (D-GaLN; cat: G1639) and dimethyl sulfoxide (DMSO; cat: D2650) were also obtained from Sigma-Aldrich (St. Louis, USA). Anti-HMGB1 (cat: ab79823), antiTLR-4 (cat: ab13867), anti-NF-אB p65 (cat: ab32536), anti-iNOS (cat: ab178945), anti-COX-2 (cat: ab179800), anti-Bcl-2 (cat: ab182858), anti-caspase-9 (cat: ab202068), and anti-caspase-3 (cat: ab184787) antibodies were obtained from Abcam (Shanghai, China).

\section{Cell Culture and Treatment}

Normal human hepatocytes (L02 cells) obtained from the Cell Bank of Type Culture Collection of the Chinese Academy of Sciences (Shanghai, China) were maintained in DMEM media (HyClone, USA; cat: SH30243.01) supplemented with 10\% (v/v) fetal bovine serum (FBS) (ExCell Bio, China; cat: FSP500), streptomycin at $37^{\circ} \mathrm{C}$ in a humidified atmosphere with $5 \%$ $\mathrm{CO}_{2}$. A Que stock solution was prepared in DMSO and diluted with culture media immediately prior to the experiment. Control cells were treated with an equal amount of DMSO alone at a final concentration of $<0.1 \%(\mathrm{v} / \mathrm{v})$.

\section{Cell Viability Assay}

To evaluate the IC50 of D-GaLN and the noncytotoxic concentration of Que on L02 cells, the effects of D-GaLN and Que on the viability of L02 cells were evaluated and counted using a Cell Counting Kit-8 (CCK-8) assay (Dojindo Laboratories, Japan; cat: CK04), according to the manufacturer's instructions. Briefly, cells were grown on 96well plates at a density of $1 \times 104$ for $12 \mathrm{~h}$. After treatment with Que and/or D-GaLN for the indicated time, the cells were incubated with $10 \mu \mathrm{l}$ of the CCK-8 solution. After incubation at $37^{\circ} \mathrm{C}$ for $2 \mathrm{~h}$ in a humidified $\mathrm{CO} 2$ incubator, the absorbance was monitored at $450 \mathrm{~nm}$ on a microplate reader (Thermo Scientific, USA). The cell viability was calculated by comparing the optical densities of samples to the control (media only) cells. The optical density of the formazan formed in control cells was taken as $100 \%$ viability.

\section{TUNEL}

The apoptotic response of L02 cells was identified using a TUNEL assay and a Fluorescein In Situ Cell Death Assay Kit (KeyGEN BioTECH, China; cat: KGA7072) according to the manufacturer's instructions. The cells were cultured in a 12 -well plate; after exposure to the desired experimental conditions, and
L02 cells were fixed with $4 \%$ paraformaldehyde (PFA) for 30 min. After washing with PBS, $0.1 \%$ Triton X-100 was allowed to permeate for $5 \mathrm{~min}$. With further washing, the reaction was carried out in a terminal deoxynucleotidyl transferase (TdT) buffer with fluorescein-labeled dUTP. The sample was then incubated with reagents at $37^{\circ} \mathrm{C}$ for $1 \mathrm{~h}$ in the dark with sealing to avoid evaporation of the reagents. After washing with PBS, the coverslips were mounted with an antifluorescent quenching sealer containing DAPI. The images were observed with a confocal laser scanning biomicroscope (Leica TCS SP8).

\section{Flow Cytometry Analysis of Apoptosis}

The ratios of apoptotic cells were measured with an Annexin VFITC/PI Apoptosis Detection Kit (KeyGEN BioTECH, China; cat: KGA108). Briefly, after exposure to the desired experimental conditions, L02 cells were collected by trypsinization and centrifugation. Then, the cells were resuspended at room temperature and fixed in a solution of a binding buffer $(195 \mu \mathrm{l}$ of annexin V-FITC, $5 \mu \mathrm{l}$ of annexin FITC, and $10 \mu \mathrm{l}$ of propidium iodide (PI)) for $15 \mathrm{~min}$ in the dark. The percentages of apoptotic cells were analyzed by flow cytometry (BD LSR Fortessa). The apoptotic rate is the apoptotic cells/ all cells.

\section{Measurement of ROS}

Intracellular ROS production was measured using an ROS assay kit (Beyotime, China; cat: S0033). The L02 cells were exposed to the desired experimental conditions, and the positive control group was incubated with Rosup for $30 \mathrm{~min}$. The cells were then incubated with $10 \mu \mathrm{M}$ CFH-DA for $30 \mathrm{~min}$ at $37^{\circ} \mathrm{C}$. Then, the cells were collected and washed with serum-free DMEM, and ROS levels were measured by flow cytometry (BD LSR Fortessa).

\section{Western Blot}

Whole cell lysis was obtained using a RIPA lysis buffer and protease inhibitor according to the user's protocol. Cytoplasmic and nuclear proteins were isolated using nuclear and cytoplasmic protein extraction kits (Beyotime, China; cat: P0028), according to the manufacturer's instructions. Protein concentration was determined using a BCA protein assay kit. An equal amount of protein $(30 \mu \mathrm{g})$ was separated by $10 \%$ to $15 \%$ SDS-PAGE and then electrotransferred onto a PVDF membrane. The membrane was blocked with $5 \%$ skim milk for $1 \mathrm{~h}$ and then incubated overnight at $4^{\circ} \mathrm{C}$ with the following antibodies: HMGB1, TLR4, caspase-3, caspase-9, Bax, Bcl-2, NF- $\kappa$ B, p65, iNOS, and COX-2. Then, the membrane was incubated with a secondary antibody for $1 \mathrm{~h}$ at room temperature. After washing 3 times with TBST, the reaction was detected with an enhanced chemiluminescent reagent (NCM Biotech, China; cat: P10100). A ImageQuantLAS4000 Chemiluminescence Imaging system was used to visualize the target proteins (GE Co., USA) and densitometry was performed using the Image J software version 1.80. Some results of western blot were presented in three technical replicates, the repeatability has been confirmed by independent experiments. 


\section{Immunofluorescence}

Cells were seeded in 12-well plates and after exposure to the desired experimental conditions, were fixed in 4\% PFA for 30 min, permeabilized in $0.5 \%$ Triton X-100 buffer for $20 \mathrm{~min}$, and blocked with 5\% BSA for $30 \mathrm{~min}$. They were then incubated with a primary antibody (rabbit anti-human HMGB1 and rabbit antihuman TLR-4) at $4^{\circ} \mathrm{C}$ overnight, with the secondary antibody (FITC/TRITC-conjugated goat anti-rabbit IgG) incubated for 1 h. After being washed with PBST, slides were covered with an anti-fluorescent quenching sealer containing DAPI. Images were observed with a confocal laser scanning biomicroscope (Leica TCS SP8).

\section{RNA Interference}

Short hairpin RNA (shRNA) was applied to silence HMGB1 at the mRNA level, as well as at the protein level. The HMGB1 shRNA (5'-GCT CAAGGAGAATTTGTAA-3') plasmid vectors (sh-HMGB1) were purchased from GeneCopoeia (Guangdong, China) and transfected into L02 cells with a negative control (shNC) using Lipofectamine ${ }^{\mathrm{TM}} 2000$ (Invitrogen, Thermo Fisher Scientific, Inc.; cat: 11668019). After transfection for $36 \mathrm{~h}$, qRT-PCR and immunoblotting assays were conducted to estimate the transfection efficiency.

\section{Real-Time PCR}

To detect the expression of HMGB1 mRNA, total RNA was extracted from the stably transfected cells using a RNAprep Pure Cell/Bacteria Kit (Tiangen, Beijing, China; cat: DP430), and cDNA was synthesized using a reverse transcriptase kit (Tiangen, Beijing, China; cat: KR116-02). The HMGB1 forward and reverse primers were $5^{\prime}$-ATATGGCAAAAGCGG ACAAG-3' and 5'-GCAACATCACCAATGGACAG- $3^{\prime}$. The $\beta$ actin forward and reverse primers were $5^{\prime}$-TGGCACCCA GCACAATGAA-3' and 5'-CTAAGTCATAGTCCGCCTAG AAGCA-3'. The melting curve data were analyzed to determine PCR specificity. Relative fold expressions were analyzed using the $2-\Delta \Delta \mathrm{Ct}$ method, using $\beta$-actin $\mathrm{Ct}$ values as the internal reference in each sample.

\section{Molecular Docking Simulations}

Molecular docking method was used to study the binding mode of Que and HMGB1. The software Ledock with Lepro tools and the web server CB-Dock were used for performing molecular docking simulations (Zhang and Zhao, 2016; Liu et al., 2019) was obtained from the RCSB Protein Data Bank and a ligand file of Que in the MOL2 was obtained from the ZINC database. For LeDock, the receptor files were processed by the LePro tool. All parameters were set to default for sampling by a combination of simulated annealing and evolutionary optimization. Docking scores were calculated by the default scoring function. Ligplot software was used for 2D interaction visualization (Laskowski and Swindells, 2011). For CB-Dock, the two files were uploaded and submitted to the CB-Dock server. The result table listed Vina scores, cavity sizes, docking centers, and sizes of predicted cavities. Once a ligand in the table is selected, the structure in the interactive $3 \mathrm{D}$ graphics is visualized.

\section{Statistical Analysis}

All experiments were repeated three times. The results were expressed as the mean \pm standard deviation (SD). GraphPad 7.0 statistical software was utilized for the statistical analyses. $p$ values were computed by ANOVA with Tukey's post hoc test. $p<0.05$ was regarded as statistically significant.

\section{RESULTS}

\section{Que Protects L02 Cells Against d-GaLN- Induced Injury}

D-GaLN is a commonly used experimental drug for causing hepatotoxic damage (Dejager and Libert, 2008; Gehrke et al., 2018). The results (Figure 1B) indicate that D-GaLN significantly reduced the viability of L02 cells in a dosedependent manner. Treatment with a concentration of 45 $\mathrm{mM} \mathrm{D}-\mathrm{GaLN}$ for $12 \mathrm{~h}$ lowered the cell viability to $52.92 \% \pm$ $5.93 \%$. Therefore, this concentration was used in subsequent experiments. To investigate the protective effects of Que, the results (Figure 1C) indicate that treatment with less than 100 $\mu \mathrm{M}$ Que did not result in significant cell death. Then, cells were pretreated with 25 to $100 \mu \mathrm{M}$ of Que for $12 \mathrm{~h}$ and then with 45 $\mathrm{mM}$ D-GaLN. The results (Figure 1D) showed that 25, 50, and $100 \mu \mathrm{M}$ of Que significantly attenuated D-GaLN-induced cell death. The protective effects of 50 and $100 \mu \mathrm{M}$ were significantly higher than those of $25 \mu \mathrm{M}$. Although there was no statistical difference, the cell viability of $50 \mu \mathrm{M}$ was higher than that of $100 \mu \mathrm{M}$. Therefore, it was determined that pretreatment with $50 \mu \mathrm{M}$ Que for $12 \mathrm{~h}$ followed by incubation with $45 \mathrm{mM}$ D-GaLN for $12 \mathrm{~h}$ was the optimal condition for the following experiments.

\section{Que Reduces L02 Cell Damage by Inhibiting ROS and Apoptosis}

We analyzed the ROS production in L02 cells by measuring the DCF fluorescence intensity. The results (Figures 2A-C) indicated that $\mathrm{D}$-GaLN significantly increased intracellular ROS accumulation, while intracellular ROS in the Que group (Figure 2D) was significantly reduced compared to the D-GaLN group.

Next, to determine the effect on apoptosis, we used a TdTmediated dUTP nick end labeling (TUNEL) assay to observe the apoptosis caused by $\mathrm{D}-\mathrm{GaLN}$ and calculate the apoptotic rates with annexin V-FITC/PI. D-GaLN significantly caused apoptosis in L02 cells (Figure 3) and increased apoptotic rates (Figures 4A, B). However, compared with the D-GaLN group, Que significantly reduced the increased apoptosis rate (Figure 4C). We further used Western blot to examine the effects of D-GaLN and Que on the expression of Bcl-2, Bax, caspase-9, and caspase3 proteins in L02 cells (Figure 5). The results show that D-GaLN significantly increased the expression of Bax, ratio of cleaved caspase- 9 and cleaved caspase-3, but decreased the expression of Bcl-2 in L02 cells. Compared with the D-GaLN group, Bax, ratio of cleaved caspase- 9 and cleaved caspase- 3 were decreased in the Que group, while Bcl-2 expression was increased. These results 


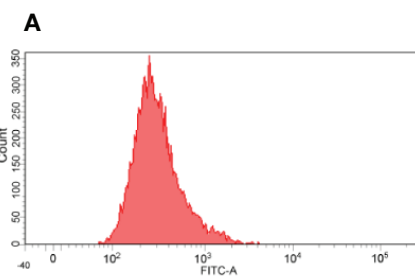

B

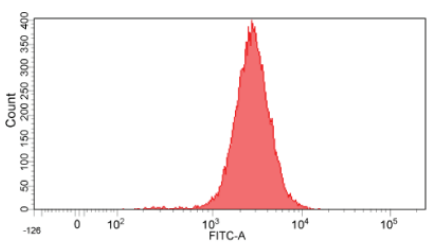

$\mathbf{F}$
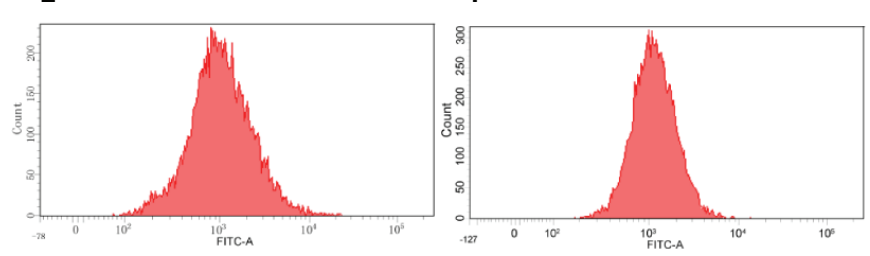

c

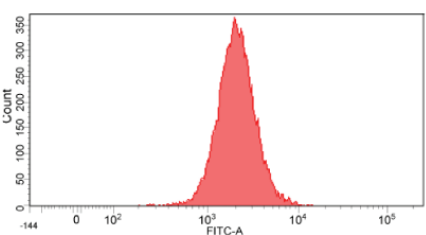

D

G

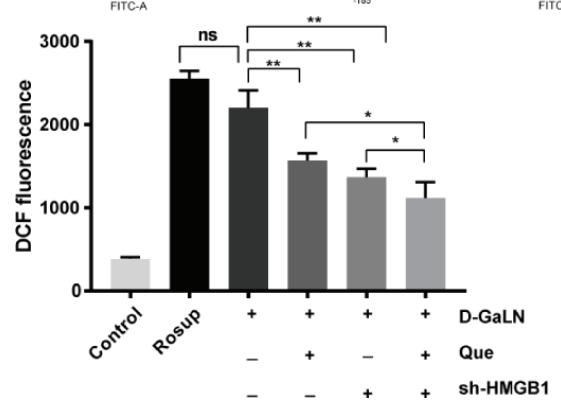

FIGURE 2 | Flow cytometric analysis of the intracellular reactive oxygen species (ROS) levels in L02 cells. (A) Control group; (B) Rosup group; (C) Cells treated with D-GaLN (45 mM) alone; (D) Cells pre-treated with Que $(50 \mu \mathrm{M})$ and then treated with D-GaLN (45 mM); (E) Cells pre-treated with sh-HMGB1 and then treated with DGaLN (45 mM); (F) Cells pre-treated with sh-HMGB1 and Que $(50 \mu \mathrm{M})$ and then treated with D-GaLN (45 mM); (G) Statistical results of ROS. Data are presented as the mean \pm SD of three independent experiments $\left({ }^{\star} p<0.05\right.$, $\left.{ }^{\star *} p<0.01\right)$; "ns" indicates not significant $(p>0.05)$.

showed that Que can weaken D-GaLN-induced oxidative stress damage and apoptosis in L02 cells.

\section{HMGB1 Aggravates d-GaLN-Induced L02 Cell Damage}

Previous studies have reported that HMGB1 aggravates damage to hepatocytes, while HMGB1 is also involved in apoptosis (Zhao et al., 2017; Wu et al., 2018). Thus, we investigated the effects of Que on HMGB1, also the expression of its receptor (TLR-4) by Western blot and immunofluorescence (IF). Figure 6 showed the increased expression of HMGB1 due to D-GaLN stimulation. Western blot also confirmed that due to the stimulation of D-GaLN, the total amount of HMGB1 and the ratio of HMGB1 in cytoplasm of L02 cells were increased. Moreover, the expression of TLR-4 in the D-GaLN group was significantly increased (Figures $\mathbf{8 B}, \mathbf{C}$ ), IF showed that TLR-4 was abundantly expressed in the cytoplasm and accumulated around the nucleus (Figure 8A). These indicated that the expression of HMGB1 was increased due to the stimulation of $\mathrm{D}-\mathrm{GaLN}$, which also affected the expression and distribution of its TLR-4 receptor.
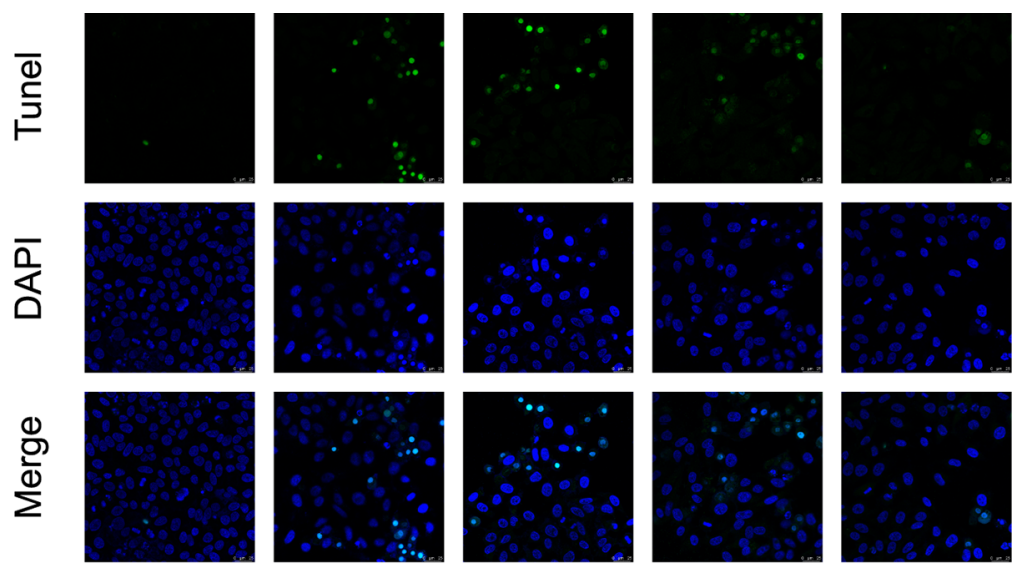

\section{D-GaLN \\ Que} sh-HMGB1
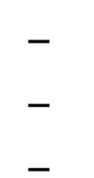

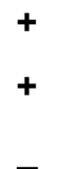

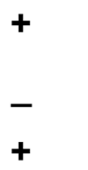

FIGURE 3 | Cell apoptosis was identified by (TdT-mediated dUTP nick end labeling) TUNEL analysis in LO2 cells. Green fluorescence represents TUNEL-positive cells. Scale bar: $25 \mu \mathrm{m}$. All nuclei were stained with 4',6-diamidino-2-phenylindole (DAPI) (blue fluorescence). 

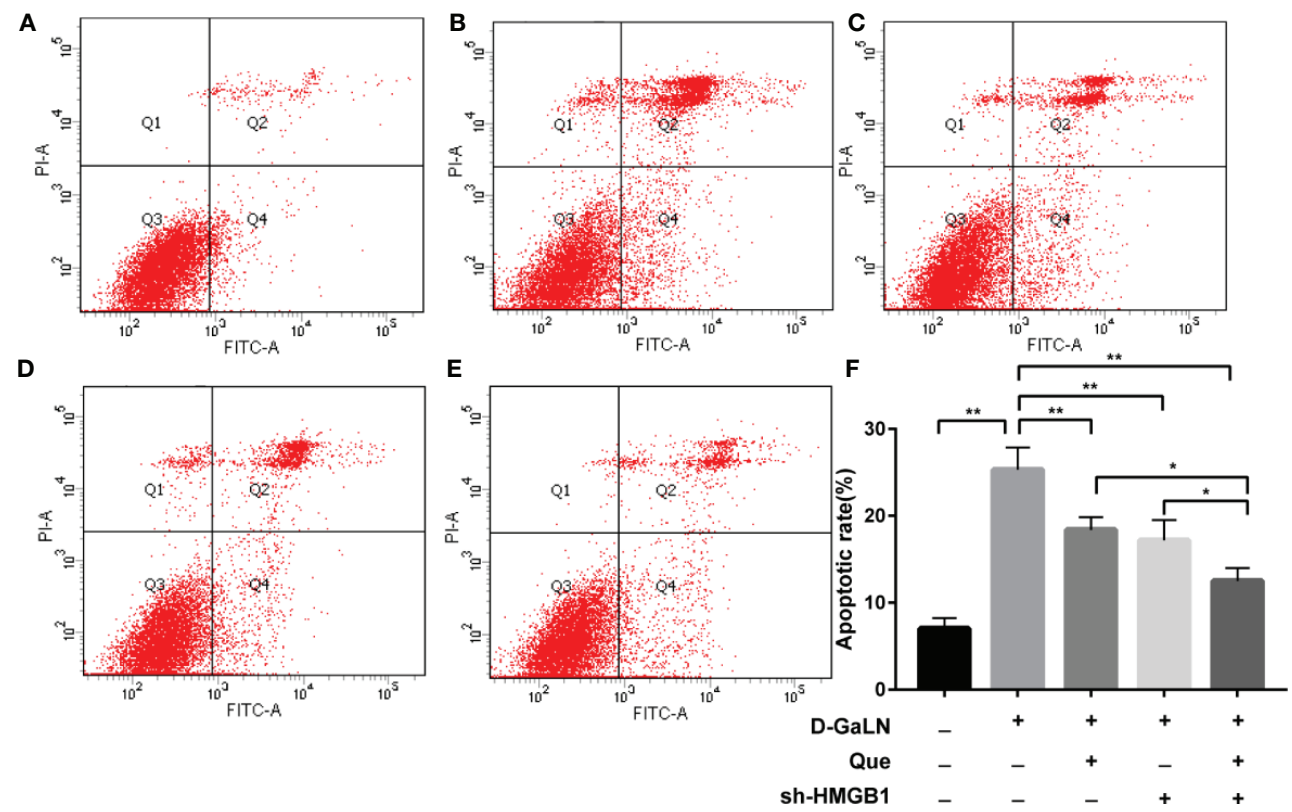

FIGURE 4 | The apoptotic rate of L02 cells was evaluated by flow cytometry after staining with annexin V-FITC/PI. (A) Control group; (B) Cells treated with D-GaLN $(45 \mathrm{mM})$ alone; (C) Cells pre-treated with Que $(50 \mu \mathrm{M})$ and then treated with D-GaLN (45 mM); (D) Cells pre-treated with sh-HMGB1 and then treated with D-GaLN $(45 \mathrm{mM}) ;$ (E) Cells pre-treated with sh-HMGB1 and Que $(50 \mu \mathrm{M})$ and then treated with D-GaLN (45 mM); (F) The results of the apoptotic rate. Data are presented as the mean \pm SD of three independent experiments $\left({ }^{*} p<0.05,{ }^{* *} p<0.01\right)$.

Thus, we investigated the function of HMGB1 in cell injury by D-GaLN. Cells were transfected with short hairpin RNAs (shRNA) targeting HMGB1. The sh-HMGB1 could achieve a reduction of $\sim 50 \%$ for HMGB1 expression (Figures $7 \mathrm{~A}, \mathbf{B}$ ). As shown in Figure 6, sh-HMGB1 downregulated the expression of
HMGB1. And the expression of TLR-4 receptors in L02 cell injury caused by D-GaLN was also decreased (Figure 8). The results of CCK-8 (Figure 7C), TUNEL (Figure 3), and flow cytometry (Figures 2E and 4D) showed that sh-HMGB1 attenuated the loss of cell viability, inhibited intracellular ROS
A

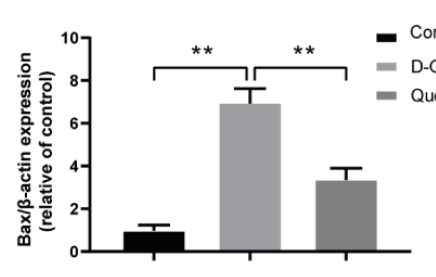

B

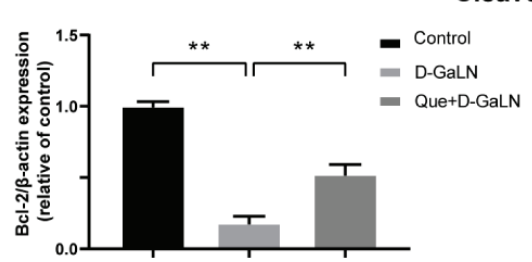

C

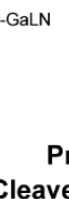

Pro-caspase-9 Cleaved-caspase-9

Pro-caspase-3

leaved-caspase-3

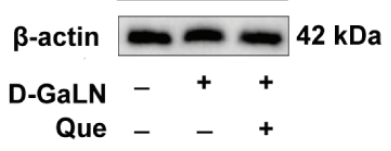

D

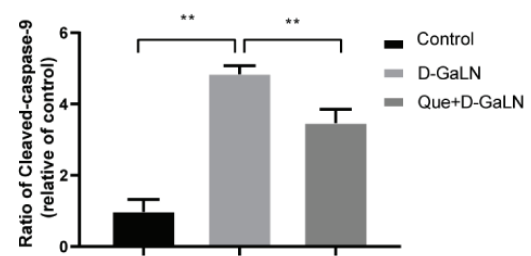

E

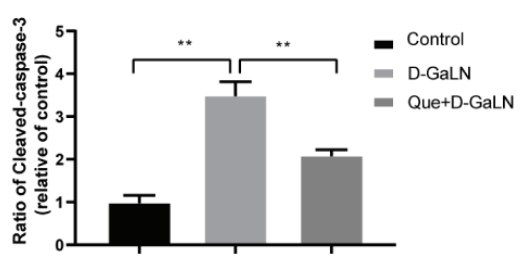

FIGURE 5 | Effect of Que on the expression of D-GaLN-induced apoptosis-related proteins. (A, B, D, E) The relative expression levels of Bax/ $\beta$-actin, Bcl-2/ $\beta$-actin, ratio of cleaved caspase-9, and ratio of cleaved caspase-3. The data on quantified protein expressions were normalized by related $\beta$-actin (fold change of control); (C) Representative immunoblots for the Bax, Bcl-2, caspase-9, caspase-3, and $\beta$-actin proteins. Data are presented as the mean \pm SD of three technical replicates $\left({ }^{* *} p<0.01\right)$. 
A
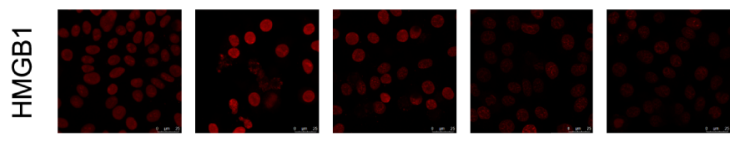

হa
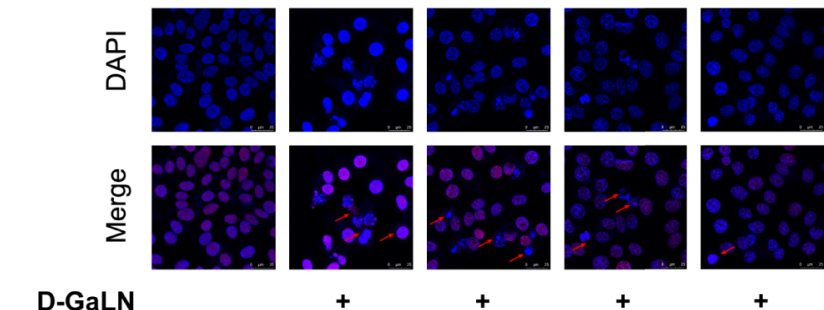

Que $-$ sh-HMGB1

B

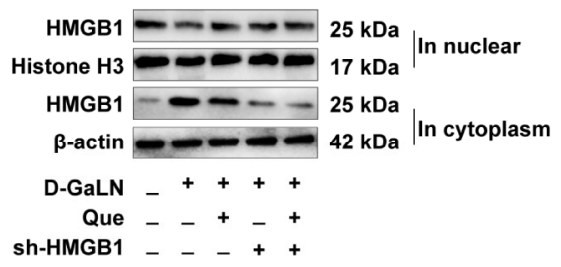

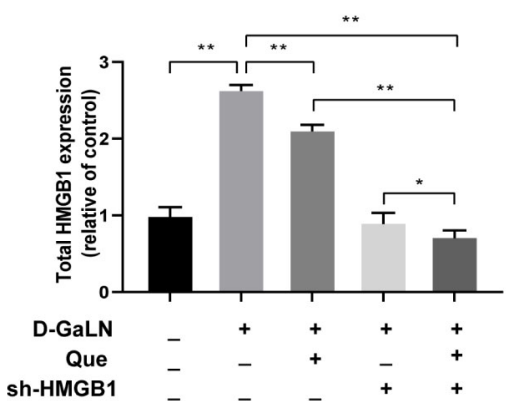

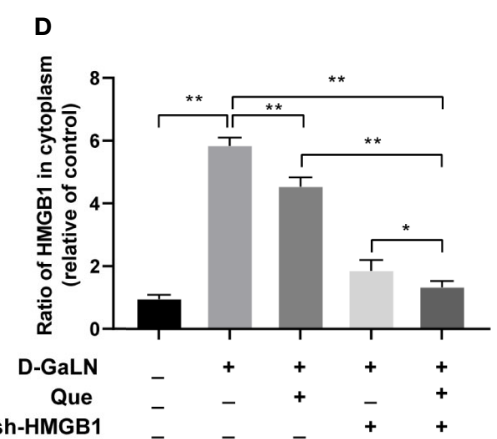

FIGURE 6 | Effect of Que on D-GaLN-induced HMGB1 expression. (A) Immunofluorescence staining of HMGB1 expression under different treatment conditions. Scale bar: $25 \mu \mathrm{m}$; Arrows indicate the HMGB1. (B) Representative immunoblots for the HMGB1 in the nucleus, Histone H3, HMGB1 in the cytoplasm, and $\beta$-actin proteins. (C, D) Total HMGB1 and ratio of HMGB1 in cytoplasm under different exposure conditions by Western blot assay. The data on HMGB1 expression in the cytoplasm were normalized by related $\beta$-actin proteins, HMGB1 in the nucleus were normalized by related Histone H3 proteins (fold change of control). Data are presented as the mean \pm SD of three technical replicates $\left({ }^{*} p<0.05,{ }^{* *} p<0.01\right)$.

accumulation and apoptosis in D-GaLN-stimulated L02 cells. Consequently, HMGB1 seemed to be related to the injury caused by $\mathrm{D}$-GaLN and may be involved in oxidative stress and apoptosis processes.

\section{Silencing of HMGB1 Enhances the Effect of Que on d-GaLN-induced L02 Cells}

The results of the CCK8 assay (Figure 7C), TUNEL (Figure 3), and flow cytometry (Figures 2F, G and 4E, F) confirmed that compared with Que and sh-HMGB1 alone, co-treatment with Que and sh-HMGB1, cell viability, ROS accumulation, and apoptosis were significantly ameliorated. We further examined changes in the expression of HMGB1 (Figure 6), when co- treated with Que and sh-HMGB1, the total amount of HMGB1 and the ratio of HMGB1 in the cytoplasm of L02 cells were decreased significantly. After silenced HMGB1, we analyzed changes in protein expression of related pathways by Western blot. Sh-HMGB1 significantly downregulated the expression of

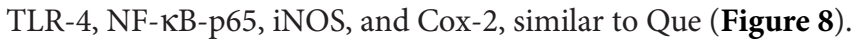
Compared with Que and D-GaLN alone, when co-treated with Que and sh-HMGB1, the inhibition of TLR-4 receptor expression and other pathway proteins were significantly enhanced (Figure 8). These results indicated that Que attenuated D-GaLN-induced L02 cell damage by suppressing ROS generation and apoptosis, which may be controlled by the inhibition of HMGB1. 
A

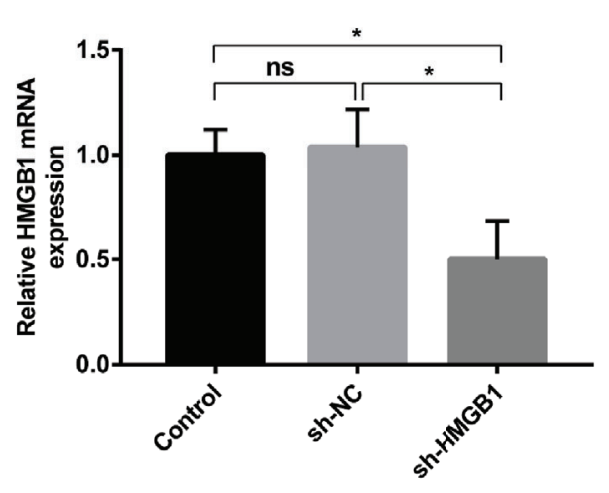

C

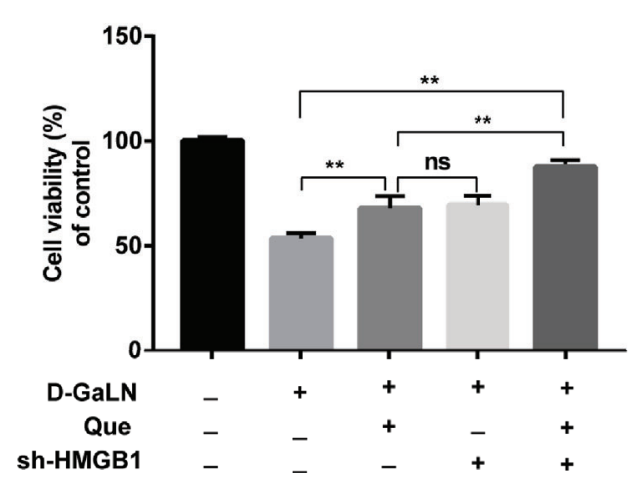

B

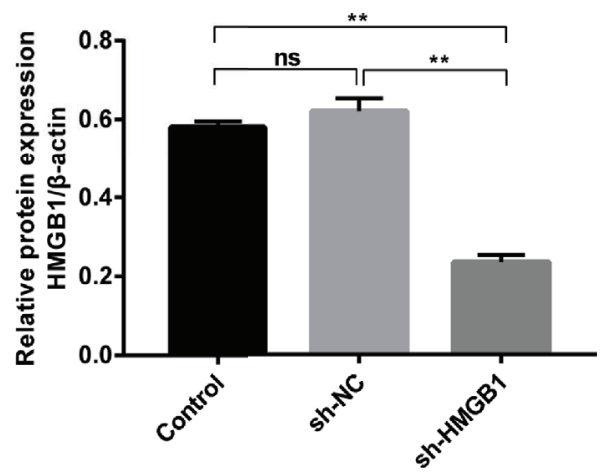

FIGURE 7 | The effect of Que on the viability of L02 cells after silenced HMGB1. The sh-HMGB1 was transfected into L02 cells, and its transfection efficiency was confirmed at (A) the mRNA level, by qRT-PCR; (B) and the protein level, by Western blot assay; (C) after silenced HMGB1, the CCK8 assay was used to analyze the cell viability of L02 cells under different exposure conditions. Data are presented as the mean $\pm \operatorname{SD}\left({ }^{*} p<0.05,{ }^{\star *} p<0.01, n=3\right)$; "ns" indicates not significant $(p>0.05)$.

\section{Structural Details of the Interaction Between HMGB1 and Que}

To better understand the molecular mechanism of Que on HMGB1, molecular docking studies on Que were performed. Molecular docking assays showed that Que can bind to the active pocket of HMGB1 crystal structure (Figure 9A) and form two stable hydrogen bonds with the Gly7 and Asp12 amino residues of HMGB1 (Figure 9B). Logarithms of free binding energy calculated by Ledock and CB-Dock were -6.23 and $-7.1 \mathrm{kcal} /$ mol. The interaction between Que and HMGB1 may affect the conformation of HMGB1, thereby inducing its downstream signal transduction.

\section{DISCUSSION}

D-GaLN is a commonly used experimental drug that causes hepatotoxicity, and its mechanism involves GSH depletion and affecting RNA synthesis (Gehrke et al., 2018). Studies have also confirmed that D-GaLN alone leads to the potent intracellular generation of ROS in HepG2 cells (Bak et al., 2018) which can induce oxidative stress in the accumulation of ROS in hepatocytes in vitro and in vivo (Gonzalez et al., 2009; Wen et al., 2018). By inducing caspase-3 activation and DNA fragmentation in hepatocytes, D-GalN causes hepatocyte apoptosis (Lin et al., 2009). Mitochondria are the source and target of ROS (Zorov et al., 2014), and excessive production of ROS leads to the apoptosis of hepatocytes (Hong et al., 2018), and D-GaLN causes apoptosis in a manner closely related to this pathway. The results of this experiment showed that D-GaLN affected the viability of L02 cells, causing ROS accumulation and mitochondrial apoptosis.

HMGB1 acts as a DAMP factor, playing an important role in various liver injuries (Geng et al., 2015). It has a significantly greater increase than chronic hepatitis, particularly in severe liver injury (Zhou et al., 2011). However, previous studies have mostly focused on the role of HMGB1 as a pro-inflammatory factor (Wang et al., 1999), 


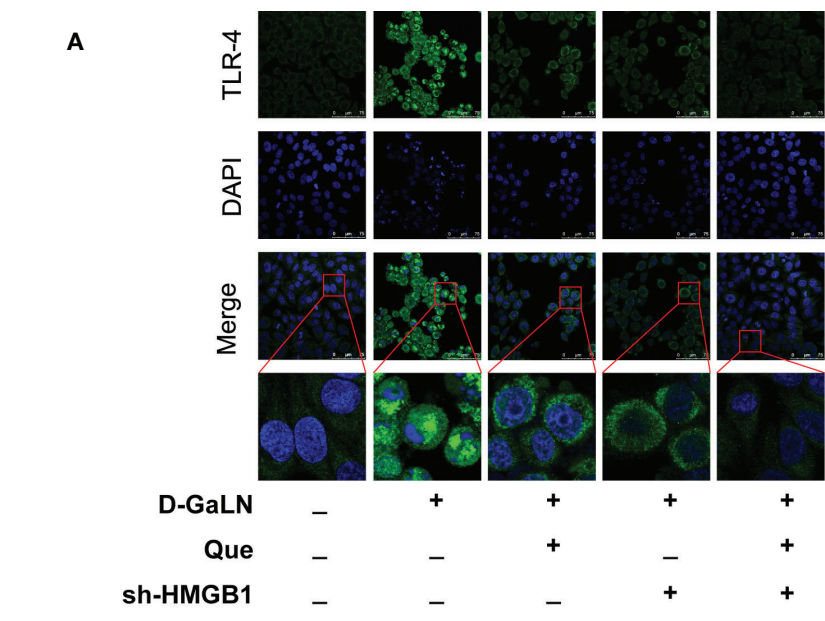

B
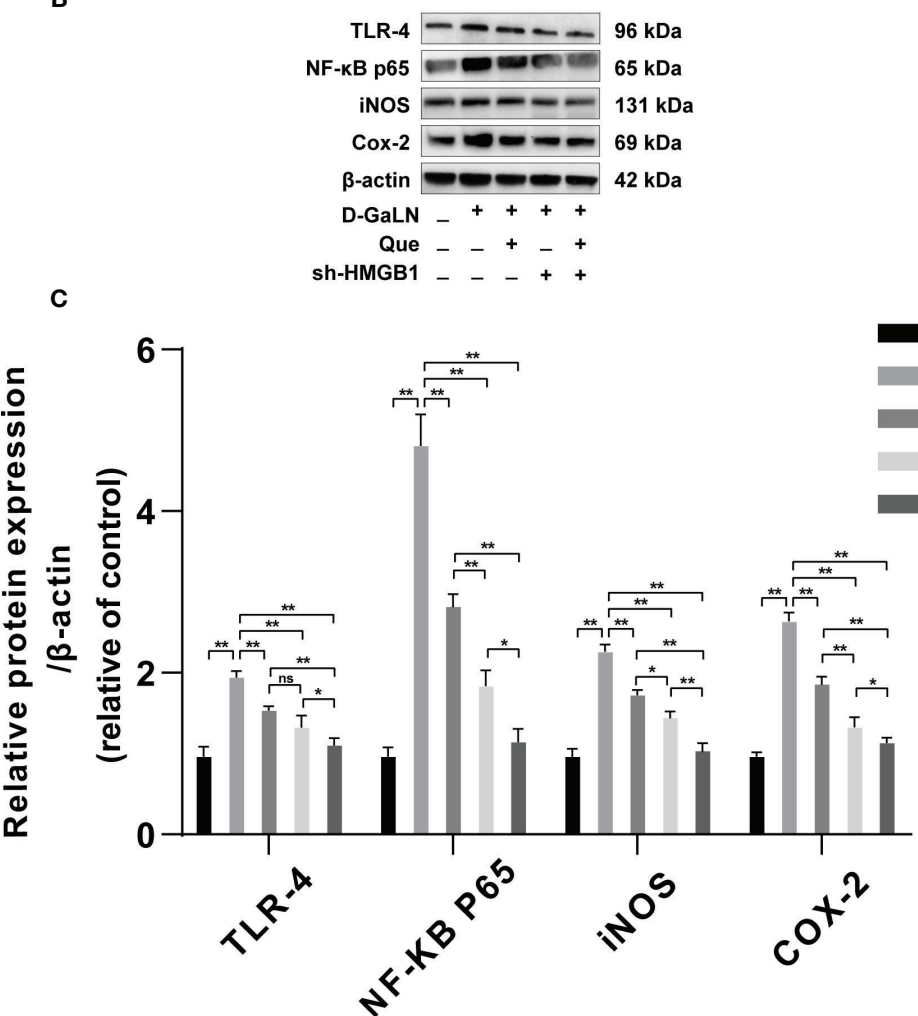

FIGURE 8 | The effect of Que on the HMGB1 signaling pathway. (A) Immunofluorescence staining of TLR-4 receptor expression under different treatment conditions. Scale bar: $75 \mu \mathrm{m}$; (B, C) the TLR-4, NF-kB P65, iNOS, and COX-2 protein expression levels were evaluated by Western blot assay. The data on quantified protein expressions were normalized by related $\beta$-actin proteins. (fold change of control). Data are presented as the mean \pm SD of three technical replicates $\left({ }^{*} p<0.05,{ }^{* \star} p<0.01\right)$.

but in addition to inflammation, excessive apoptosis is also an important mechanism of cell death in liver failure (Komarov et al., 2016). Blocking HMGB1 can inhibit caspase-3 activation, thereby reducing apoptosis (Tan et al., 2018). Recent studies have shown that apoptotic cells activate macrophages to release HMGB1 (Velegraki et al., 2013). HMGB1 interacts with phosphatidylserine on the surface of apoptotic neutrophils, thereby inhibiting the clearance of neutrophils by macrophage phagocytic cells (Liu et al., 2008). By binding to DNA, late-stage apoptotic cells can 
A

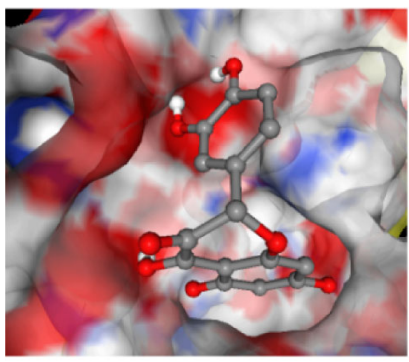

B

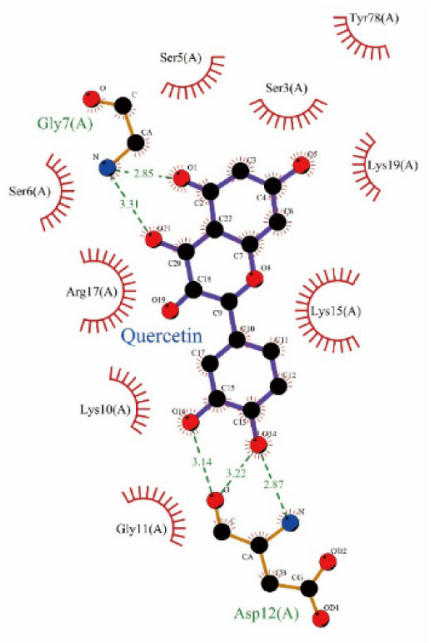

HMGB1

FIGURE 9 | The structural details of the interaction between HMGB1 and Que were obtained by the docking method. (A) Surface representation of HMGB1-Que complex. (B) The interaction zone with Que showed the residues of interactions.

release HMGB1 (Shi et al., 2018); the release of HMGB1 was also present in cells with late apoptosis (Pisetsky, 2014) After being released, caspase- 3 can be activated by the HMGB1TLR4 pathway, resulting in apoptosis (Zhang et al., 2019).

Meanwhile, there is also evidence to confirm that HMGB1 is essential for oxidative stress (Tang et al., 2011). In vitro, recombinant HMGB1 leads to the TLR-4-dependent activation of NADPH oxidase and increased ROS production (Zhang et al., 2015). TLR-4-dependent ROS production and calcium-mediated signaling are involved in the HMGB1 release induced by liver ischemia (Tsung et al., 2007). Further, the mitochondria play an important role in apoptosis by relocating intermembrane mitochondrial proteins, such as Bcl-2 and Bax (Bhola and Letai, 2016). The induction of ROS can regulate mitochondrial membrane potential, leading to apoptosis initiation in the mitochondrial pathway (Sinha et al., 2013). Therefore, we hypothesize that HMGB1-mediated apoptosis is caused by the mitochondrial release of apoptotic proteins caused by ROS. To the best of our knowledge, the results of this experiment have demonstrated, for the first time, that HMGB 1 participated in D-GaLN-induced L02 cell injury. In the injured L02 cells, the expression of its TLR-4 receptor and signaling pathway factors also increased accordingly. Moreover, after silenced HMGB1, ROS production and apoptosis were significantly improved. Therefore, HMGB1 is closely related to the occurrence of oxidative stress and mitochondrial apoptosis.

Que is a commonly used dietary supplement flavonoid (D'Andrea, 2015); it can alleviate acute liver injury induced by lipopolysaccharide (LPS)/D-GalN through anti-inflammatory, antioxidative, and anti-apoptotic activity (He et al., 2019). Que also prevents oleic acid-induced ROS production and mitochondrial damage in HepG2 hepatocytes (Rafiei et al., 2019) and can protect against oxidative stress by inhibiting the iNOS/NF- $\kappa \mathrm{B}$ pathway (Bahar et al., 2017). Meanwhile, Que is also a potential inhibitor of HMGB1 (Musumeci et al., 2014). In this experiment, we demonstrated that Que protects L02 cells from damage caused by D-GaLN. Moreover, we confirmed for the first time that the protective effect of Que on L02 cells is produced by inhibiting HMGB1 and subsequent oxidative stress and mitochondrial apoptosis mediated by the associated signaling pathway. And molecular docking showed that the high affinity of Que and HMGB1 is related to the hydrogen bonding with Gly7 and Asp12 residues. This binding may affect the conformation of HMGB1, thereby inducing its downstream signal transduction.

\section{CONCLUSION}

In conclusion, our current results indicate that HMGB1 was involved in D-GaLN-induced L02 cell injury. However, Que can inhibit HMGB1 to protect L02 cells from D-GaLN-mediated damage. This protective effect is associated with the inhibition of oxidative stress and mitochondrial apoptosis mediated by HMGB1. Further, molecular docking showed that hydrogen bonding with Gly7 and Asp12 residues are involved in the binding of Que and HMGB1. Therefore, our experimental results provide a theoretical basis for using Que as a hepatoprotective drug targeting HMGB1. 


\section{DATA AVAILABILITY STATEMENT}

All datasets generated for this study are included in the article/ supplementary material.

\section{AUTHOR CONTRIBUTIONS}

PF and QZ conceived and designed the experiments. JL, XW, XJ, $\mathrm{XF}, \mathrm{MW}, \mathrm{XW}, \mathrm{WY}$, and $\mathrm{WH}$ were involved in the experimental study design, preparation, and review of this manuscript. All

\section{REFERENCES}

Bahar, E., Kim, J. Y., and Yoon, H. (2017). Quercetin Attenuates ManganeseInduced Neuroinflammation by Alleviating Oxidative Stress through Regulation of Apoptosis, iNOS/NF-kappaB and HO-1/Nrf2 Pathways. Int. J. Mol. Sci. 18 (9), 1989. doi: 10.3390/ijms18091989

Bai, Y., Du, S., Li, F., Huang, F., Deng, R., Zhou, J., et al. (2017). Histone deacetylase-high mobility group box-1 pathway targeted by hypaconitine suppresses the apoptosis of endothelial cells. Exp. Biol. Med. (Maywood) 242 (5), 527-535. doi: 10.1177/1535370216685433

Bak, D. H., Na, J., Choi, M. J., Lee, B. C., Oh, C. T., Kim, J. Y., et al. (2018). Antiapoptotic effects of human placental hydrolysate against hepatocyte toxicity in vivo and in vitro. Int. J. Mol. Med. 42 (5), 2569-2583. doi: 10.3892/ijmm.2018.3830

Bhola, P. D., and Letai, A. (2016). Mitochondria-Judges and Executioners of Cell Death Sentences. Mol. Cell 61 (5), 695-704. doi: 10.1016/j.molcel.2016.02.019

D'Andrea, G. (2015). Quercetin: A flavonol with multifaceted therapeutic applications? Fitoterapia 106, 256-271. doi: 10.1016/j.fitote.2015.09.018

Darband, S. G., Kaviani, M., Yousefi, B., Sadighparvar, S., Pakdel, F. G., Attari, J. A., et al. (2018). Quercetin: A functional dietary flavonoid with potential chemopreventive properties in colorectal cancer. J. Cell Physiol. 233 (9), 6544-6560. doi: $10.1002 /$ jcp. 26595

de Oliveira, M. R., Nabavi, S. M., Braidy, N., Setzer, W. N., Ahmed, T., and Nabavi, S. F. (2016). Quercetin and the mitochondria: A mechanistic view. Biotechnol. $A d v .34$ (5), 532-549. doi: 10.1016/j.biotechadv.2015.12.014

Dejager, L., and Libert, C. (2008). Tumor necrosis factor alpha mediates the lethal hepatotoxic effects of poly(I:C) in D-galactosamine-sensitized mice. Cytokine 42 (1), 55-61. doi: 10.1016/j.cyto.2008.01.014

Gehrke, N., Hovelmeyer, N., Waisman, A., Straub, B. K., Weinmann-Menke, J., Worns, M. A., et al. (2018). Hepatocyte-specific deletion of IL1-RI attenuates liver injury by blocking IL-1 driven autoinflammation. J. Hepatol. 68 (5), 986995. doi: 10.1016/j.jhep.2018.01.008

Geng, Y., Ma, Q., Liu, Y. N., Peng, N., Yuan, F. F., Li, X. G., et al. (2015). Heatstroke induces liver injury via IL-1beta and HMGB1-induced pyroptosis. J. Hepatol. 63 (3), 622-633. doi: 10.1016/j.jhep.2015.04.010

Gonzalez, R., Ferrin, G., Hidalgo, A. B., Ranchal, I., Lopez-Cillero, P., SantosGonzalez, M., et al. (2009). N-acetylcysteine, coenzyme Q10 and superoxide dismutase mimetic prevent mitochondrial cell dysfunction and cell death induced by d-galactosamine in primary culture of human hepatocytes. Chem. Biol. Interact. 181 (1), 95-106. doi: 10.1016/j.cbi.2009.06.003

He, Y., Xia, Z., Yu, D., Wang, J., Jin, L., Huang, D., et al. (2019). Hepatoprotective effects and structure-activity relationship of five flavonoids against lipopolysaccharide/d-galactosamine induced acute liver failure in mice. Int. Immunopharmacol. 68, 171-178. doi: 10.1016/ j.intimp.2018.12.059

Hernandez, C., Huebener, P., Pradere, J. P., Antoine, D. J., Friedman, R. A., and Schwabe, R. F. (2018). HMGB1 links chronic liver injury to progenitor responses and hepatocarcinogenesis. J. Clin. Invest. 128 (6), 2436-2451. doi: 10.1172/JCI91786

Hong, H., Wu, H., Chen, J., Wu, B., Yu, H., Yan, B., et al. (2018). Cytotoxicity induced by iodinated haloacetamides via ROS accumulation and apoptosis in authors have reviewed and approved the final version of the manuscript.

\section{FUNDING}

This research was funded by the National Natural Science Foundation of China (Grant no. 81573767), Natural Science Foundation of Beijing Municipality (Grant no. 7192024).
HepG-2cells. Environ. Pollut. 242 (Pt A), 191-197. doi: 10.1016/ j.envpol.2018.06.090

Hu, Y. B., Hu, D. P., and Fu, R. Q. (2017). Correlation between high mobility group box-1 protein and chronic hepatitis $\mathrm{B}$ infection with severe hepatitis $\mathrm{B}$ and acute-on-chronic liver failure: a meta-analysis. Minerva Med. 108 (3), 268 276. doi: 10.23736/S0026-4806.16.04865-5

Huebener, P., Pradere, J. P., Hernandez, C., Gwak, G. Y., Caviglia, J. M., Mu, X., et al. (2015). The HMGB1/RAGE axis triggers neutrophil-mediated injury amplification following necrosis. J. Clin. Invest. 125 (2), 539-550. doi: 10.1172/ JCI76887

Kang, R., Chen, R., Zhang, Q., Hou, W., Wu, S., Cao, L., et al. (2014). HMGB1 in health and disease. Mol. Aspects Med. 40, 1-116. doi: 10.1016/ j.mam.2014.05.001

Klotz, L. O., and Steinbrenner, H. (2017). Cellular adaptation to xenobiotics: Interplay between xenosensors, reactive oxygen species and FOXO transcription factors. Redox Biol. 13, 646-654. doi: 10.1016/j.redox.2017.07.015

Komarov, A. P., Komarova, E. A., Green, K., Novototskaya, L. R., Baker, P. S Eroshkin, A., et al. (2016). Functional genetics-directed identification of novel pharmacological inhibitors of FAS- and TNF-dependent apoptosis that protect mice from acute liver failure. Cell Death Dis. 7, e2145. doi: 10.1038/ cddis. 2016.45

Laskowski, R. A., and Swindells, M. B. (2011). LigPlot+: multiple ligand-protein interaction diagrams for drug discovery. J. Chem. Inf. Model 51 (10), 27782786. doi: 10.1021/ci200227u

Li, X., Liu, H. C., Yao, Q. Y., Xu, B. L., Zhang, S. C., and Tu, C. T. (2016). Quercetin Protects Mice from ConA-Induced Hepatitis by Inhibiting HMGB1-TLR Expression and Down-Regulating the Nuclear Factor Kappa B Pathway. Inflammation 39 (1), 96-106. doi: 10.1007/s10753-015-0227-9

Li, X., Jin, Q., Yao, Q., Xu, B., Li, L., Zhang, S., et al. (2018). The Flavonoid Quercetin Ameliorates Liver Inflammation and Fibrosis by Regulating Hepatic Macrophages Activation and Polarization in Mice. Front. Pharmacol. 9, 72. doi: 10.3389/fphar.2018.00072

Lin, B. R., Yu, C. J., Chen, W. C., Lee, H. S., Chang, H. M., Lee, Y. C., et al. (2009). Green tea extract supplement reduces D-galactosamine-induced acute liver injury by inhibition of apoptotic and proinflammatory signaling. J. BioMed. Sci. 16, 35. doi: 10.1186/1423-0127-16-35

Liu, G., Wang, J., Park, Y. J., Tsuruta, Y., Lorne, E. F., Zhao, X., et al. (2008). High mobility group protein-1 inhibits phagocytosis of apoptotic neutrophils through binding to phosphatidylserine. J. Immunol. 181 (6), 4240-4246. doi: 10.4049/jimmunol.181.6.4240

Liu, Y., Grimm, M., Dai, W. T., Hou, M. C., Xiao, Z. X., and Cao, Y. (2019). CBDock: a web server for cavity detection-guided protein-ligand blind docking. Acta Pharmacol. Sin. 41 (1), 138-144. doi: 10.1038/s41401-019-0228-6

Miltonprabu, S., Tomczyk, M., Skalicka-Wozniak, K., Rastrelli, L., Daglia, M., Nabavi, S. F., et al. (2017). Hepatoprotective effect of quercetin: From chemistry to medicine. Food Chem. Toxicol. 108 (Pt B), 365-374. doi: 10.1016/j.fct.2016.08.034

Musumeci, D., Roviello, G. N., and Montesarchio, D. (2014). An overview on HMGB1 inhibitors as potential therapeutic agents in HMGB1-related pathologies. Pharmacol. Ther. 141 (3), 347-357. doi: 10.1016/ j.pharmthera.2013.11.001 
Petrovic, A., Bogojevic, D., Korac, A., Golic, I., Jovanovic-Stojanov, S., Martinovic, V., et al. (2017). Oxidative stress-dependent contribution of HMGB1 to the interplay between apoptosis and autophagy in diabetic rat liver. J. Physiol. Biochem. 73 (4), 511-521. doi: 10.1007/s13105-017-0574-0

Pisetsky, D. S. (2014). The translocation of nuclear molecules during inflammation and cell death. Antioxid. Redox Signal 20 (7), 1117-1125. doi: 10.1089/ars.2012.5143

Rafiei, H., Omidian, K., and Bandy, B. (2019). Phenolic Breakdown Products of Cyanidin and Quercetin Contribute to Protection against Mitochondrial Impairment and Reactive Oxygen Species Generation in an In Vitro Model of Hepatocyte Steatosis. J. Agric. Food Chem. 67 (22), 6241-6247. doi: 10.1021/ acs.jafc.9b02367

Scaffidi, P., Misteli, T., and Bianchi, M. E. (2002). Release of chromatin protein HMGB1 by necrotic cells triggers inflammation. Nature 418 (6894), 191-195. doi: 10.1038 /nature00858

Shi, Y., Guo, X., Zhang, J., Zhou, H., Sun, B., and Feng, J. (2018). DNA binding protein HMGB1 secreted by activated microglia promotes the apoptosis of hippocampal neurons in diabetes complicated with OSA. Brain Behav. Immun. 73, 482-492. doi: 10.1016/j.bbi.2018.06.012

Sinha, K., Das, J., Pal, P. B., and Sil, P. C. (2013). Oxidative stress: the mitochondria-dependent and mitochondria-independent pathways of apoptosis. Arch. Toxicol. 87 (7), 1157-1180. doi: 10.1007/s00204-013-1034-4

Tan, J. Y., Zhao, F., Deng, S. X., Zhu, H. C., Gong, Y., and Wang, W. (2018). Glycyrrhizin affects monocyte migration and apoptosis by blocking HMGB1 signaling. Mol. Med. Rep. 17 (4), 5970-5975. doi: 10.3892/mmr.2018.8598

Tang, D., Kang, R., Zeh, H., and Lotze, M. T. (2011). High-mobility group box 1, oxidative stress, and disease. Antioxid. Redox Signal 14 (7), 1315-1335. doi: 10.1089/ars.2010.3356

Tsung, A., Klune, J. R., Zhang, X., Jeyabalan, G., Cao, Z., Peng, X., et al. (2007). HMGB1 release induced by liver ischemia involves Toll-like receptor 4 dependent reactive oxygen species production and calcium-mediated signaling. J. Exp. Med. 204 (12), 2913-2923. doi: 10.1084/jem.20070247

Velegraki, M., Papakonstanti, E., Mavroudi, I., Psyllaki, M., Tsatsanis, C., Oulas, A., et al. (2013). Impaired clearance of apoptotic cells leads to HMGB1 release in the bone marrow of patients with myelodysplastic syndromes and induces TLR4-mediated cytokine production. Haematologica 98 (8), 1206-1215. doi: 10.3324/haematol.2012.064642

Wang, H., Bloom, O., Zhang, M., Vishnubhakat, J. M., Ombrellino, M., Che, J., et al. (1999). HMG-1 as a late mediator of endotoxin lethality in mice. Science 285 (5425), 248-251. doi: 10.1126/science.285.5425.248

Wang, R., Zhang, H., Wang, Y., Song, F., and Yuan, Y. (2017). Inhibitory effects of quercetin on the progression of liver fibrosis through the regulation of NF-small ka, CyrillicB/Ismall ka, CyrillicBalpha, p38 MAPK, and Bcl-2/Bax signaling. Int. Immunopharmacol. 47, 126-133. doi: 10.1016/j.intimp.2017.03.029

Wen, J., Lin, H., Zhao, M., Tao, L., Yang, Y., Xu, X., et al. (2018). Piceatannol attenuates D-GalN/LPS-induced hepatoxicity in mice: Involvement of ER stress, inflammation and oxidative stress. Int. Immunopharmacol. 64, 131139. doi: 10.1016/j.intimp.2018.08.037

Wu, H., Chen, Z., Chen, J. Z., Pei, L. G., Xie, J., Wei, Z. H., et al. (2018). High Mobility Group B-1 (HMGB-1) Promotes Apoptosis of Macrophage-Derived Foam Cells by Inducing Endoplasmic Reticulum Stress. Cell Physiol. Biochem. 48 (3), 1019-1029. doi: 10.1159/000491970

Yamamoto, T., and Tajima, Y. (2017). HMGB1 is a promising therapeutic target for acute liver failure. Expert Rev. Gastroenterol. Hepatol. 11 (7), 673-682. doi: 10.1080/17474124.2017.1345625
Yang, R., Zou, X., Tenhunen, J., and Tonnessen, T. I. (2017). HMGB1 and Extracellular Histones Significantly Contribute to Systemic Inflammation and Multiple Organ Failure in Acute Liver Failure. Mediators Inflammation 2017, 5928078. doi: 10.1155/2017/5928078

Zhang, N., and Zhao, H. (2016). Enriching screening libraries with bioactive fragment space. Bioorg. Med. Chem. Lett. 26 (15), 3594-3597. doi: 10.1016/ j.bmcl.2016.06.013

Zhang, C., Mo, M., Ding, W., Liu, W., Yan, D., Deng, J., et al. (2014). Highmobility group box 1 (HMGB1) impaired cardiac excitation-contraction coupling by enhancing the sarcoplasmic reticulum (SR) $\mathrm{Ca}(2+)$ leak through TLR4-ROS signaling in cardiomyocytes. J. Mol. Cell Cardiol. 74, 260-273. doi: 10.1016/j.yjmcc.2014.06.003

Zhang, Y., Karki, R., and Igwe, O. J. (2015). Toll-like receptor 4 signaling: A common pathway for interactions between prooxidants and extracellular disulfide high mobility group box 1 (HMGB1) protein-coupled activation. Biochem. Pharmacol. 98 (1), 132-143. doi: 10.1016/j.bcp.2015.08.109

Zhang, M., Hu, X., Li, S., Lu, C., Li, J., Zong, Y., et al. (2018). Hepatoprotective effects of ethyl pyruvate against $\mathrm{CCl} 4$-induced hepatic fibrosis via inhibition of TLR4/NF-kappaB signaling and up-regulation of MMPs/ TIMPs ratio. Clin. Res. Hepatol. Gastroenterol. 42 (1), 72-81. doi: 10.1016/ j.clinre.2017.04.008

Zhang, Y., Zhang, H., Zhang, Z., Li, S., Jiang, W., Li, X., et al. (2019). LncRNA MALAT1 cessation antagonizes hypoxia/reoxygenation injury in hepatocytes by inhibiting apoptosis and inflammation via the HMGB1-TLR4 axis. Mol. Immunol. 112, 22-29. doi: 10.1016/j.molimm.2019.04.015

Zhao, G., Fu, C., Wang, L., Zhu, L., Yan, Y., Xiang, Y., et al. (2017). Downregulation of nuclear $\mathrm{HMGB} 1$ reduces ischemia-induced HMGB1 translocation and release and protects against liver ischemia-reperfusion injury. Sci. Rep. 7, 46272. doi: 10.1038/srep46272

Zheng, Y. Z., Deng, G., Liang, Q., Chen, D. F., Guo, R., and Lai, R. C. (2017). Antioxidant Activity of Quercetin and Its Glucosides from Propolis: A Theoretical Study. Sci. Rep. 7 (1), 7543. doi: 10.1038/s41598-017-08024-8

Zhou, R. R., Zhao, S. S., Zou, M. X., Zhang, P., Zhang, B. X., Dai, X. H., et al. (2011). HMGB1 cytoplasmic translocation in patients with acute liver failure. BMC Gastroenterol. 11, 21. doi: 10.1186/1471-230X-11-21

Zorov, D. B., Juhaszova, M., and Sollott, S. J. (2014). Mitochondrial reactive oxygen species (ROS) and ROS-induced ROS release. Physiol. Rev. 94 (3), 909950. doi: 10.1152/physrev.00026.2013

Zou, W., Liu, W., Yang, B., Wu, L., Yang, J., Zou, T., et al. (2015). Quercetin protects against perfluorooctanoic acid-induced liver injury by attenuating oxidative stress and inflammatory response in mice. Int. Immunopharmacol. 28 (1), 129-135. doi: 10.1016/j.intimp.2015.05.043

Conflict of Interest: The authors declare that the research was conducted in the absence of any commercial or financial relationships that could be construed as a potential conflict of interest.

Copyright (c) 2020 Fang, Liang, Jiang, Fang, Wu, Wei, Yang, Hou and Zhang. This is an open-access article distributed under the terms of the Creative Commons Attribution License (CC BY). The use, distribution or reproduction in other forums is permitted, provided the original author(s) and the copyright owner(s) are credited and that the original publication in this journal is cited, in accordance with accepted academic practice. No use, distribution or reproduction is permitted which does not comply with these terms. 